\title{
Layered Steered Space-Time Codes Using Multi-Dimensional Sphere Packing Modulation
}

\author{
Mohammed El-Hajjar, Graduate Student Member, IEEE, Osamah Alamri, Jin Wang, Member, IEEE, \\ Salam Zummo, Member, IEEE, and Lajos Hanzo, Fellow, IEEE
}

\begin{abstract}
We present a novel multi-functional Multiple-Input Multiple-Output (MIMO) scheme, that combines the benefits of Space-Time Codes (STC), of Vertical Bell Labs Layered SpaceTime (V-BLAST) scheme as well as of beamforming. To further enhance the attainable system performance and to maximise the coding advantage of the proposed transmission scheme, the system is also combined with multi-dimensional Sphere Packing (SP) modulation. Additionally, we quantify the capacity of the proposed multi-functional MIMO aided multi-dimensional SP arrangement and propose a novel technique of computing an upper limit on the achievable bandwidth efficiency of the system based on EXtrinsic Infomation Transfer (EXIT) charts. Further system performance improvements can be attained by serially concatenating our proposed scheme with an outer code together with a Unity-Rate Code (URC), where three different receiver structures are created by varying the iterative detection configuration of the constituent decoders/demappers. Moreover, the convergence behaviour of the proposed schemes is evaluated with the aid of EXIT charts. Explicitly, the three proposed systems are capable of operating within $0.9 \mathrm{~dB}, 0.6 \mathrm{~dB}$ and $0.4 \mathrm{~dB}$ of the maximum achievable rate limit. Additionally, the three stage assisted SP aided scheme is capable of outperforming its counterpart employing QPSK by 1 dB at a BER of $10^{-6}$.
\end{abstract}

Index Terms-Layered space-time codes; steered space-time codes; beam-forming; beam-steering; multi-functional MIMO; MIMO; sphere-packing modulation; multi-dimensional modulation; turbo detection, iterative detection; VBLAST; EXIT-charts; wireless channel capacity.

\section{INTRODUCTION}

$\mathbf{M}$ ULTIPLE-INPUT Multiple-Output (MIMO) systems attain a higher capacity than single-input single-output systems [1-3]. In [4] the multi-layer MIMO structure known as the Vertical Bell Labs Layered Space-Time (V-BLAST) scheme was proposed, whose receiver is capable of providing a high multiplexing gain without the need for any increase in the transmitted power or in the system's bandwidth. On the other hand, Space-Time Block Codes (STBC) [5] are capable of achieving the maximum possible diversity gain, while using low-complexity linear processing at the receiver. Hence, it was proposed in [6] to combine the benefits of these two techniques for the sake of providing both antenna diversity as well as spectral efficiency gains. This hybrid scheme was improved in [7] by optimising the decoding order

Manuscript received November 19, 2007; accepted January 16, 2008. The associate editor coordinating the review of this letter and approving it for publication was A. Chockalingam.

The financial support of Vodafone under the auspices of the Dorothy Hodgkin Postgraduate Award and that of the European Union as well as the support of EPSRC, UK is gratefully acknowledged.

The authors are with the School of Electrical and Computer Science, Univ. of Southampton, United Kingdom (e-mail: lh@ecs.soton.ac.uk).

Digital Object Identifier 10.1109/TWC.2009.071292 of the different antenna layers. Furthermore, beamforming [8] constitutes an effective technique of reducing the multipleaccess interference, where the antenna gain is increased in the direction of the desired user. Additionally, beamforming has been combined with STBC to attain a higher Signal-to-Noise Ratio (SNR) gain [9].

In [10] Su et al. demonstrated that Sphere Packing (SP) aided STBC was capable of outperforming the conventional STBC scheme dispensing with SP [5]. In [11] the SP demapper of [10] was modified for the sake of accepting the $a$ priori information passed to it from the channel decoder as extrinsic information.

It was demonstrated in [12] that a recursive inner code is needed in order to avoid the formation of a Bit Error Ratio (BER) floor, when employing iterative decoding. In [13], rate1 inner codes were employed for designing low complexity turbo codes suitable for bandwidth and power limited systems having stringent BER requirements. EXtrinsic Information Transfer (EXIT) charts were introduced in [14] for characterising the flow of extrinsic information between the soft-in softout constituent decoders. The concept of EXIT chart analysis has been extended to three-stage concatenated systems in [15].

The rationale of the paper can be summarised as follows. We amalgamate the merits of V-BLAST, STC and beamforming for the sake of achieving a high multiplexing gain, a high diversity gain as well as beamforming gain. The resultant scheme is referred to here as a Layered Steered SpaceTime Code (LSSTC). Additionally, the system is combined with multi-dimensional SP modulation, which is capable of maximising the coding advantage of the transmission scheme. Moreover, the optimum bit-to-SP-symbol mapper is designed using an EXIT-chart based procedure allowing us to achieve diverse design objectives.

On the other hand, the novelty of the paper can be outlined as follows. We quantify the capacity of the LSSTC-SP scheme for transmission over both Rayleigh as well as Gaussian channels. Furthermore, we propose a novel technique for quantifying the maximum achievable rate of the system using EXIT charts. We also propose three turbo-detected LSSTC-SP receiver structures, where iterative detection is carried out between an outer code's Decoder I, an intermediate code's Decoder II and an LSSTC-SP demapper. A comparison between the three turbo-detected schemes reveals that a carefully designed two-stage iterative detection scheme is capable of operating sufficiently close to capacity at a lower complexity, when compared to a three-stage system employing a Recursive Systematic Convolutional (RSC) code or a two-stage system employing Irregular Convolutional Code (IrCC) as an outer 


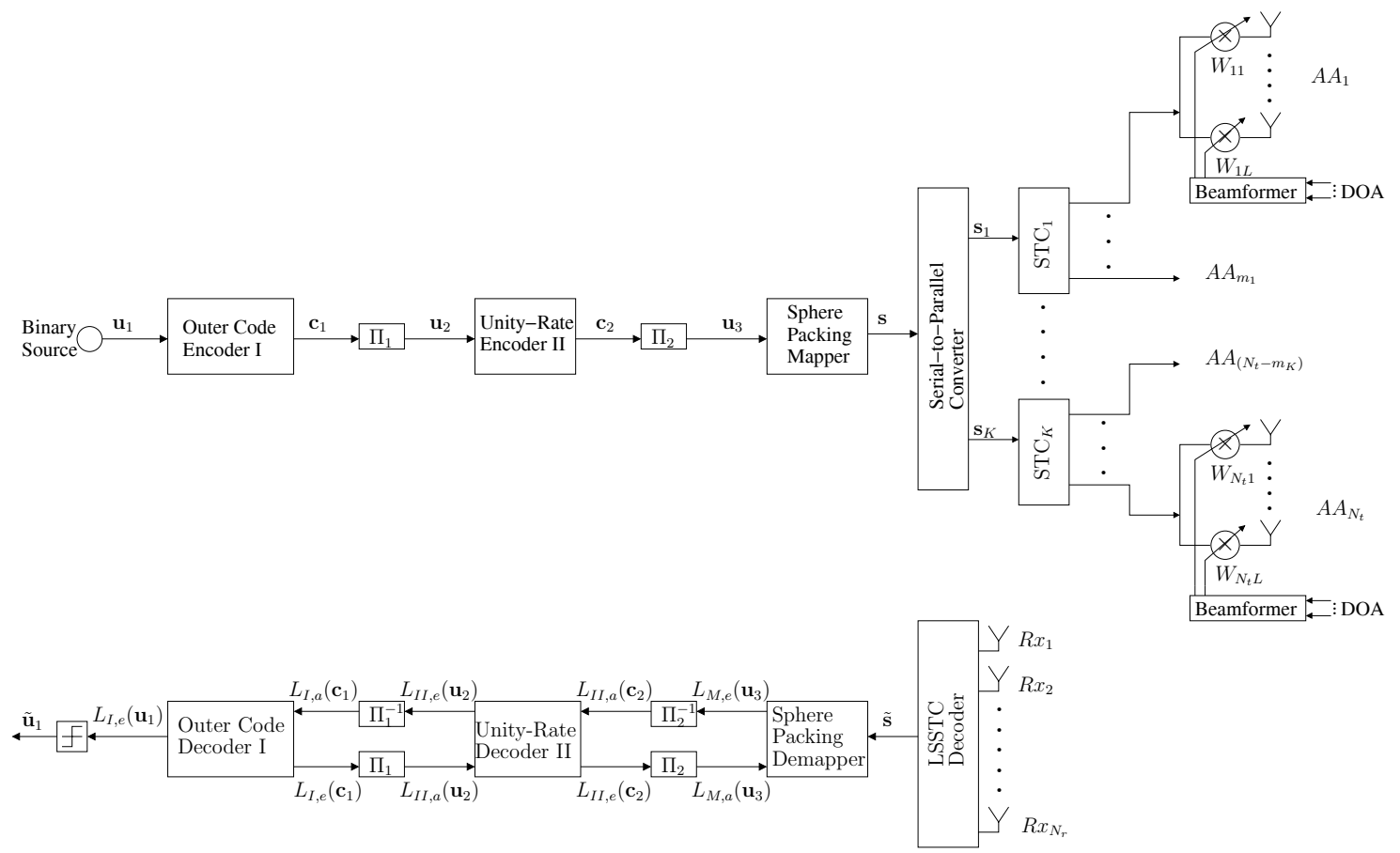

Fig. 1. Block diagram of the LSSTC aided iteratively-detected SP modulation.

code.

In Section II we present the encoding and decoding algorithms of the novel LSSTC scheme and demonstrate how the scheme can be combined with multi-dimensional SP modulation. In Section III we quantify the capacity of the LSSTC-SP scheme, followed by a discussion of the iterative detection schemes invoked in Section IV. Finally, the attainable performance of these schemes is studied comparatively in Section V, followed by our conclusion in Section VI.

\section{Layered Steered Space-Time Codes using SPHERE PaCKING MOdULATION}

\section{A. Transmitter Model}

A block diagram of the proposed LSSTC scheme is illustrated in Figure 1. The antenna architecture employed in Figure 1 has $N_{t}$ transmit Antenna Arrays (AA) spaced sufficiently far apart in order to experience independent fading and hence to achieve transmit diversity. The $L$ number of elements of each of the AAs are spaced at a distance of $d=\lambda / 2$ for the sake of achieving a beamforming gain, where $\lambda$ represents the carrier's wavelength. Furthermore, the receiver is equipped with $N_{r} \geqslant N_{t}$ antennas. According to Figure 1, a block of $B$ input information symbols "s" is serial-to-parallel converted to $K$ groups of symbol streams of length $B_{1}, B_{2}, \cdots, B_{K}$, where $B_{1}+B_{2}+\cdots+B_{K}=B$. Each group of $B_{k}$ symbols, $k \in[1, K]$, is then encoded by a component space-time code $\mathrm{STC}_{k}$ associated with $m_{k}$ transmit AAs, where $m_{1}+m_{2}+\cdots+m_{K}=N_{t}$. In this contribution, we consider transmissions over a temporally correlated narrowband Rayleigh fading channel associated with a normalised Doppler frequency of 0.01 .

The $L$-dimensional Spatio-Temporal Channel Impulse Response (CIR) vector spanning the $m$ th transmitter AA, $m \in$ $\left[1, \cdots, N_{t}\right]$, and the $n$th receiver antenna, $n \in\left[1, \cdots, N_{r}\right]$, can be expressed as

$$
\begin{aligned}
\mathbf{h}_{n m}(t) & =\mathbf{a}_{n m}(t) \delta\left(t-\tau_{k}\right) \\
& =\left[a_{n m, 0}(t), \ldots, a_{n m,(L-1)}(t)\right]^{T} \delta\left(t-\tau_{k}\right),(1)
\end{aligned}
$$

where $\tau_{k}$ is the signal's delay and $a_{n m, l}(t)$ is the CIR with respect to the $m n$th link and the $l$ th element of the $m$ th AA. Based on the assumption that the array elements are separated by half a wavelength, we have $\mathbf{a}_{n m}(t)=\alpha_{n m}(t) \cdot \mathbf{d}_{n m}$, where $\alpha_{n m}(t)$ is a Rayleigh faded envelope, $\mathbf{d}_{n m}=[1$, $\left.\exp \left(j\left[\pi \sin \left(\psi_{n m}\right)\right]\right), \ldots, \exp \left(j\left[(L-1) \pi \sin \left(\psi_{n m}\right)\right]\right)\right]^{T}$ and $\psi_{n m}$ is the $n m$ th link's Direction Of Arrival (DOA).

\section{B. Receiver Model}

The received baseband data matrix $\mathbf{Y}$ can be expressed as $\mathbf{Y}=\mathbf{H W X}+\mathbf{N}$, where $\mathbf{X}$ represents the transmitted STC symbols matrix, $\mathbf{H}$ is an $\left(N_{r} \times N_{t}\right)$ matrix whose entries of $\mathbf{h}_{n m}$ are defined in Equation (1) and $\mathbf{N}$ denotes the AWGN matrix whose entries have a variance of $N_{0} / 2$ per dimension. Furthermore, $\mathbf{W}$ is a diagonal AA weight matrix, whose diagonal entry $\mathbf{w}_{m n}$ is the $L$-dimensional weight vector for the $m$ th beamformer AA and the $n$th receive antenna. In this scenario, the MRC criterion based transmit beamformer, which constitutes an effective solution to maximising the antenna gain, is the optimum beamformer. Let $\mathbf{w}_{m n}=\mathbf{d}_{n m}^{\dagger}$, where the superscript ${ }^{\dagger}$ represents the conjugate transpose operation. Then the received signal can be expressed as $\mathbf{Y}=L \hat{\mathbf{H X}}+\mathbf{N}$, where $\widehat{\mathbf{H}}$ is an $\left(N_{r} \times N_{t}\right)$ matrix, whose entries are $\alpha_{n m}$. Moreover, $\mathbf{Y}$ can be written as $\mathbf{Y}=L \sum_{k=1}^{K} \widehat{\mathbf{H}}_{k} \mathbf{x}_{k}+\mathbf{N}$, where $\mathbf{x}_{k}$ represents the component STC used at layer $k$, with $k \in[1, \cdots, K]$.

The most beneficial decoding order of the STC layers is determined on the basis of detecting the highest-power layer 


$$
C_{D C M C}=\max _{p\left(\mathcal{S}^{1}\right), \cdots, p\left(\mathcal{S}^{M}\right)} \sum_{i=1}^{M} \underbrace{\int_{-\infty}^{\infty} \ldots \int_{-\infty}^{\infty}}_{D \text {-fold }} p\left(\tilde{\mathcal{S}} \mid \mathcal{S}_{i}\right) \cdot p\left(\mathcal{S}_{i}\right) \cdot \log _{2}\left(\frac{p\left(\tilde{\mathcal{S}} \mid \mathcal{S}_{i}\right)}{\sum_{v=1}^{M} p\left(\tilde{\mathcal{S}} \mid \mathcal{S}_{v}\right) \cdot p\left(\mathcal{S}_{v}\right)}\right) \cdot d \tilde{\mathcal{S}} \quad[\mathrm{bit} / \mathrm{sym}] .
$$

first for the sake of a high correct detection probability. For simplicity, let us consider the case of $K=2$ STBC layers and that layer 1 is detected first. The decoder computes an orthonormal basis for the left null space of $\widehat{\mathbf{H}}_{2}$ and assigns the vectors of the basis to the rows of a matrix $\mathbf{Q}$, so that $\mathbf{Q} \cdot \widehat{\mathbf{H}}_{2}=0$. Multiplying $\mathbf{Q}$ by $\mathbf{Y}$ suppresses the interference imposed on layer 1, followed by direct STBC detection. Then, the decoder subtracts the remodulated contribution of the decoded symbols of layer 1 from the composite twin-layer received signal Y. Finally, the decoder applies direct STBC decoding to the second layer, since the interference imposed by the first layer has been eliminated. This group-interference cancellation procedure can be generalised to any $N_{t}$ and $K$ values.

\section{LSSTC and SP modulation}

According to the previous discussion, the decoded signal represents a scaled version of the transmitted signal corrupted by AWGN. This observation implies that the diversity product of the LSSTC scheme is determined by the minimum Euclidean distance of all legitimate transmitted vectors. Our idea is to jointly design the legitimate $m_{k}$-component complexvalued vectors $\left(x_{1}, x_{2}, \cdots, x_{m_{k}}\right)$, so that they are represented by a single phasor point selected from a SP constellation corresponding to a $2 m_{k}$-dimensional real-valued lattice having the best known minimum Euclidean distance in the real-valued space $\mathbb{R}^{2 m_{k}}$.

In this contribution we assume that each layer is constituted by a twin-AA STBC scheme, i.e. $m_{k}=2, \forall k \in[0, K]$, which means that the SP design required is $2 m_{k}=4$-dimensional. Assuming that there are $L_{s p}$ legitimate vectors $\left(x_{l, 1}, x_{l, 2}\right)$, $l=0,1, \ldots, L_{s p}-1$, the transmitter then has to choose the modulated signal from these $L_{s p}$ legitimate symbols to be transmitted over the two AAs. Assuming that $\mathbf{S}=$ $\left\{\mathbf{s}^{l}=\left[a_{l, 1}, a_{l, 2}, a_{l, 3}, a_{l, 4}\right] \in \mathbb{R}^{4}: 0 \leq l \leq L_{s p}-1\right\}$ constitutes a set of $L_{s p}$ legitimate constellation points from the lattice $\mathbb{D}_{4}$ [16], upon introducing the notation $\mathbf{C}_{l}=\left\{x_{l, 1}, x_{l, 2}\right\}=$ $\left\{a_{l, 1}+j a_{l, 2}, a_{l, 3}+j a_{l, 4}\right\}$, we have a set of constellation symbols leading to the design of LSSTC signals, whose diversity product is determined by the minimum Euclidean distance of the set of $L_{s p}$ legitimate constellation points in $\mathbf{S}$.

\section{CAPACITY OF LSSTC-SP SCHEME}

Upon using the decoding order of $(1,2, \cdots, K)$, group $k$ will have a diversity order of $m_{k} \times\left(N_{r}-N_{t}+m_{1}+m_{2}+\right.$ $\left.\cdots+m_{k}\right)=m_{k} \times N_{r k}$. Thus, the received sphere-packed symbol of layer $k$, assuming perfect interference cancellation, can be described as $\tilde{\mathbf{s}}_{k}=\sum_{r=1}^{N_{r k}} \chi_{2 m_{k} r}^{2} \mathbf{s}_{k}+\Delta_{k}$, where $\chi_{2 m_{k} r}^{2}=L \sum_{t=1}^{m_{k}} \alpha_{r t}$ represents a chi-squared distributed random variable having $2 m_{k}$ degrees of freedom and $\Delta_{k} \in \mathbb{R}^{4}$ is the AWGN after decoding having a covariance matrix of $\sigma_{\Delta_{k}}^{2} \cdot \mathbf{I}_{4}=\chi_{2 m_{k, r}}^{2} \cdot N_{0} / 2 \cdot \mathbf{I}_{4}$.
Let $\tilde{\mathcal{S}}=\left(\tilde{\mathbf{s}}_{1}, \tilde{\mathbf{s}}_{2}, \cdots, \tilde{\mathbf{s}}_{K}\right)^{T}, \mathcal{S}=\left(\mathbf{s}_{1}, \mathbf{s}_{2}, \cdots, \mathbf{s}_{K}\right)^{T}$ and note that in conjunction with $K$ groups there are $M=\left(L_{s p}\right)^{K}$ number of possible SP phasor combinations. Thus, the achievable capacity of the LSSTC scheme for transmission over the Discrete-Input Continuous-Output Memoryless Channel (DCMC) can be derived from that of the discrete memoryless channel according to Equation $(2)[17,18]$, where $p\left(\mathcal{S}_{i}\right)$ is the probability of occurrence for the transmitted symbol vector $\mathcal{S}_{i}$. Furthermore, since the components of the vectors $\mathcal{S}$ and $\tilde{\mathcal{S}}$ are independent, we have $p(\tilde{\mathcal{S}} \mid \mathcal{S})=\prod_{k=1}^{K} p\left(\tilde{\mathbf{s}}_{k} \mid \mathbf{s}_{k}\right)$.

Moreover, $C_{D C M C}$ in Equation (2) is maximised, when the transmitted symbols are equiprobably distributed, i.e. for $p\left(\mathcal{S}_{i}\right)=\frac{1}{M}[18]$. Hence, we can write

$$
\begin{aligned}
& \log _{2}\left(\frac{p\left(\tilde{\mathcal{S}} \mid \mathcal{S}_{i}\right)}{\sum_{v=1}^{M} p\left(\tilde{\mathcal{S}} \mid \mathcal{S}_{v}\right) \cdot p\left(\mathcal{S}_{v}\right)}\right)= \\
& \log _{2}(M)-\log _{2}\left(\sum_{v=1}^{M} \prod_{k=1}^{K} \exp \left(\Psi_{k, v i}\right)\right),
\end{aligned}
$$

where the term $\Psi_{k, v i}$ is given by

$$
\begin{aligned}
& \Psi_{k, v i}= \\
& \sum_{d=1}^{D=4} \sum_{r=1}^{N_{r k}}\left(-\frac{\left(\chi_{2 m_{k} r}^{2}[d]\left(\mathbf{s}_{k, i}[d]-\mathbf{s}_{k, v}[d]\right)+\Delta_{i}[d]\right)^{2}}{\chi_{2 m_{k} r}^{2}[d] \cdot N_{0}}\right. \\
& \left.+\frac{\left(\Delta_{i}[d]\right)^{2}}{\chi_{2 m_{k} r}^{2}[d] \cdot N_{0}}\right) .
\end{aligned}
$$

Finally, Equation (2) can be simplified to

$$
\begin{gathered}
C_{D C M C}=\log _{2}(M) \\
-\frac{1}{M} \sum_{i=1}^{M} \prod_{k=1}^{K} E\left[\log _{2}\left(\sum_{v=1}^{M} \prod_{k=1}^{K} \exp \left(\Psi_{k, v i}\right)\right) \mid \mathbf{s}_{i}\right],
\end{gathered}
$$

where $E[A \mid B]$ is the expectation of A conditioned on $\mathrm{B}$.

On the other hand, the Continuous-Input ContinuousOutput Memoryless Channel (CCMC) capacity of the proposed LSSTC scheme can be expressed as $C_{C C M C}=$ $\sum_{k=1}^{K} E\left[\frac{D}{2} \log _{2}\left(1+\sum_{r=1}^{N_{r k}} \chi_{2 m_{k}, r}^{2} \frac{S N R}{N_{t}}\right)\right] \quad[\mathrm{bit} / \mathrm{sym}] \quad[17$, 18]. Finally, the bandwidth efficiency is related to the capacity according to $\eta=\frac{C}{D / 2}$ in $[\mathrm{bits} / \mathrm{sec} / \mathrm{Hz}]$, where $D$ is the dimension of the constellation used [18].

\section{Iterative Detection AND EXIT ChaRt ANALysis}

The block diagram of the iteratively-detected LSSTC-aided SP modulation is shown in Figure 1. In this contribution we present three iterative detection aided SP assisted LSSTC schemes. The first and second systems, referred to as System 1 and System 2 respectively, employ no iterations between the Unity-Rate Code (URC) Decoder II and the SP demapper of Figure 1. However, the two systems differ in the choice of the 


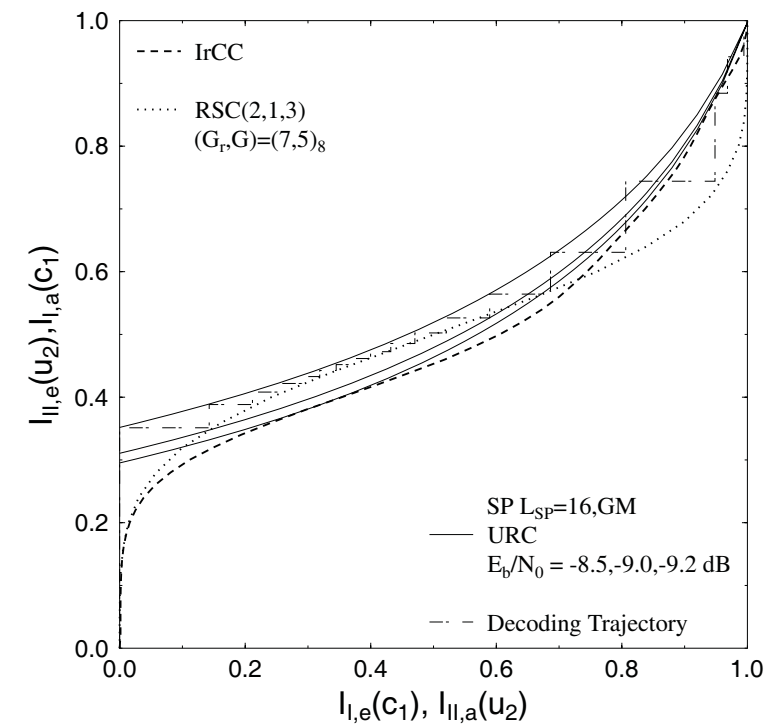

Fig. 2. EXIT chart of the proposed LSSTC-SP aided System 1 and System 2 employing iterative detection between a $1 / 2$-rate $\mathrm{RSC} / \mathrm{IrCC}$ decoder and a URC decoder employing Gray mapping aided SP in conjunction with $L_{s p}=$ 16 , while using an interleaver length of $D=180,000$ bits.

outer Encoder I, while System 1 employs a regular RSC code, System 2 uses an IrCC. Finally, System 3 invokes three-stage iterative detection exchanging extrinsic information between the SP demapper, the URC Decoder II, and the outer RSC Decoder I. In what follows, all the results presented characterise an LSSTC-SP scheme using $\left(N_{t}, N_{r}\right)=(4,4), L=4$ elements per AA, $K=2$ and $L_{s p}=16$.

In the two-stage iterative detectors of System 1 and System 2, the EXIT characteristics of Decoders I and II can be described by the following two EXIT functions, $I_{I, e}\left(\mathbf{c}_{1}\right)=T_{I, c_{1}}\left[I_{I, a}\left(\mathbf{c}_{1}\right)\right]$ and $I_{I I, e}\left(\mathbf{u}_{2}\right)=$ $T_{I I, u_{2}}\left[I_{I I, a}\left(\mathbf{u}_{2}\right), E_{b} / N_{0}\right]$ [14], where $I .(x)$ denotes the mutual information (MI) between the corresponding LLRs and the bits $x$, while the subscripts $a$ and $e$ denote $a$ priori and extrinsic information, respectively.

Figure 2 shows the EXIT chart of System 1 employing a turbo-detection aided channel-coded LSSTC-SP system using Gray Mapping (GM). The main objective of employing EXIT charts [14] is to predict the convergence behaviour of the iterative decoder by examining the evolution of the input/output MI exchange between the constituent decoders. The system employs a rate $R_{I}=1 / 2$ memory- 2 RSC code in conjunction with octal generator polynomials of $\left(G_{r}, G\right)=(7,5)_{8}$, where $G_{r}$ is the feedback polynomial. Encoder II is a simple URC scheme, described by the pair of octal generator polynomials $\left(G, G_{r}\right)=(2,3)_{8}$. The Gray Mapping (GM) of the bits to SP symbols was used because no iterations are invoked between the SP demapper and the decoders, and in this case a higher initial MI and hence a higher starting point is achieved at $I_{A}=0$ in the EXIT curve of Figure 2, where an open convergence tunnel is formed around $E_{b} / N_{0}=-8.5 \mathrm{~dB}$. The EXIT chart based convergence predictions can be verified by the Monte-Carlo simulation based iterative decoding trajectory of Figure 2, where the trajectory was recorded at $E_{b} / N_{0}=-8.5 \mathrm{~dB}$, while using an interleaver length of $D=180,000$ bits.

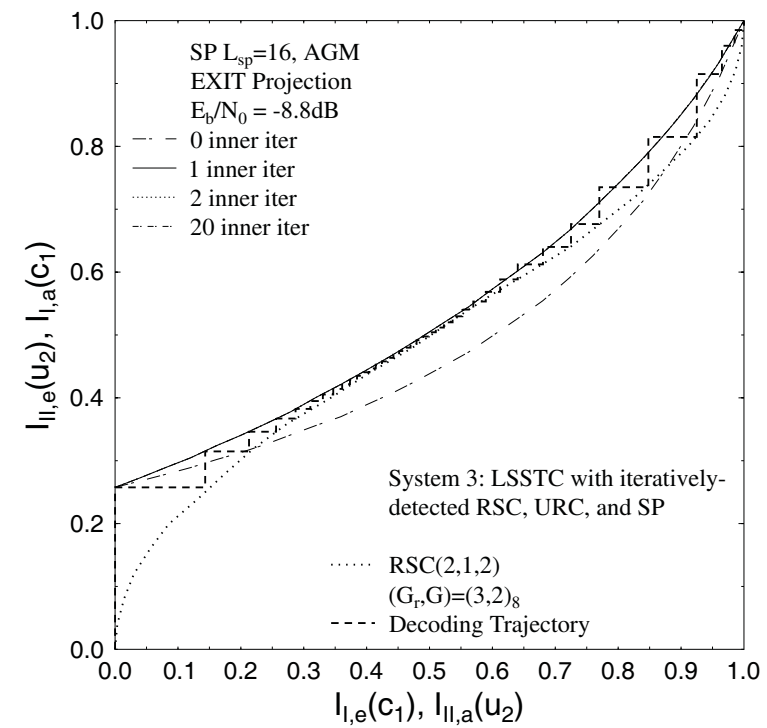

Fig. 3. 2D projection of the EXIT charts of the three-stage RSC-coded LSSTC-SP scheme, when employing anti-Gray mapping aided SP in conjunction with $L_{s p}=16$, while using an interleaver length of $D=180,000$ bits.

On the other hand, it is a well-understood property of the conventional 2D EXIT charts that a narrow but marginally open EXIT-tunnel represents a near-capacity performance [19, 20]. Therefore, in System 2 we invoke IrCCs for the sake of appropriately shaping the EXIT curves by minimising the area within the EXIT-tunnel using the procedure of $[19,21]$. Figure 2 also shows the EXIT chart of System 2 employing a turbo-detection aided channel-coded LSSTC-SP system using $\mathrm{GM}$, where the iterations are carried out between the outer $1 / 2$-rate IrCC and the inner URC decoders. Observe from Figure 2 that an open convergence tunnel is formed around $E_{b} / N_{0}=-9.2 \mathrm{~dB}$. This implies that according to the predictions of the EXIT chart of Figure 2, the iterative decoding process is expected to converge at $E_{b} / N_{0}=-9.2 \mathrm{~dB}$.

On the other hand, in System 3 the extrinsic information is exchanged between the SP demapper, the URC Decoder II and the outer Decoder I. As seen from Figure 1, the input of Decoder II is constituted by the a priori input $L_{I I, a}\left(\mathbf{c}_{2}\right)$ and the a priori input $L_{I I, a}\left(\mathbf{u}_{2}\right)$ after appropriately ordering the data provided by the SP demapper and Decoder I, respectively. Therefore, the EXIT characteristics of Decoder II can be represented by a three dimensional EXIT chart. However, it is cumbersome to interpret the 3D EXIT charts. Hence, we only show the 2D representation of the corresponding EXIT charts. Figure 3 shows the 2D-projected EXIT curve of the combined SP demapper and Decoder II at $E_{b} / N_{0}=-8.8 \mathrm{~dB}$, when employing the best possible ${ }^{1}$ Anti-Gray Mapping ${ }^{2}$ (AGM). According to Figure 3, when no inner iterations are carried out between the SP demapper and Decoder II, the system requires $E_{b} / N_{0}>-8.8 \mathrm{~dB}$ for maintaining an open tunnel. However, observe that when $I=1$, 2 and 20 inner iterations are carried out, the open EXIT tunnel

\footnotetext{
${ }^{1}$ The best possible AGM was found by evaluating the achievable performance of all AGM schemes.

${ }^{2}$ Anti-Gray Mapping (AGM) is used here to refer to any non-Gray mapping scheme.
} 


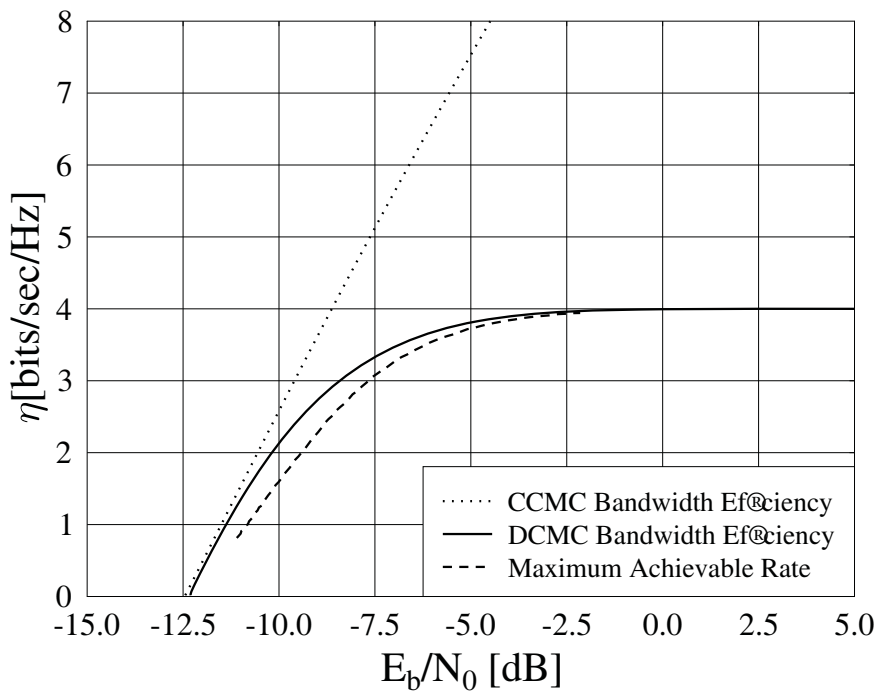

Fig. 4. Capacity of the four transmit antenna arrays aided LSSTC-SP system in conjunction with $L_{s p}=16$ employing four elements per AA for both DCMC and CCMC together with the maximum achievable rate obtained using EXIT charts.

is formed at $E_{b} / N_{0}=-8.8 \mathrm{~dB}$. Therefore, in our results we use a single inner iteration that produces the same result and imposes the lowest complexity. The EXIT chart prediction of Figure 3, namely that an infinitesimally low BER may be attained beyond $E_{b} / N_{0}=-8.8 \mathrm{~dB}$, is confirmed by the decoding trajectory of Figure 3, which was recorded for an interleaver depth of $D=180,000$ bits.

\section{A. Maximum Achievable Rate}

An upper limit on the maximum achievable rate of the system can be calculated based on the area property of $\overline{\mathcal{A}}_{I} \approx R_{I}$, where $\overline{\mathcal{A}}_{I}$ is the inverse of the area under the EXIT-curve of Decoder I. More explicitly, it was observed in [20,21] that the outer Decoder I may have a maximum rate of $R_{I}^{\max } \approx \mathcal{A}_{I I}$ at a specific $E_{b} / N_{0}$ value, where $\mathcal{A}_{I I}$ is the area under the EXIT curve of the inner decoder. Therefore, if $\mathcal{A}_{I I}$ is calculated for different $E_{b} / N_{0}$ values, the maximum achievable bandwidth efficiency in bits/sec/Hz may be formulated as a function of the $E_{b} / N_{0}$ value as follows

$$
\begin{aligned}
\eta_{\max }\left(\underline{\left.E_{b} / N_{0}\right)}\right. & =B_{s p} \cdot R_{L S S T C-S P} \cdot R_{I}^{\max } \\
& \approx B_{s p} \cdot R_{L S S T C-S P} \cdot \mathcal{A}_{I I}\left(E_{b} / N_{0}\right),
\end{aligned}
$$

where $B_{s p}=\log _{2}\left(L_{s p}\right)$ is the number of bits per SP symbol and $R_{L S S T C-S P}=1$ for the system employing the parameters outlined at the beginning of Section IV, since $T=2$ time slots are needed to transmit two SP symbols. Additionally, $\underline{E_{b} / N_{0}}$ and $E_{b} / N_{0}$ are related to each other as follows $\underline{E_{b} / N_{0}=E_{b}} / N_{0}+10 \log \left(\frac{R_{o}}{\mathcal{A}_{I I}\left(E_{b} / N_{0}\right)}\right)[\mathrm{dB}]$, where $R_{o}$ is the original outer code rate used when generating the EXIT curves.

The MIMO channel's capacity curves recorded for the 4dimensional SP modulation assisted LSSTC scheme in conjunction with $\left(N_{t}, N_{r}\right)=(4,4)$ and $L=4$ elements per AA are shown in Figure 4, portraying both the DCMC and CCMC capacity curves as well as the maximum achievable rate of

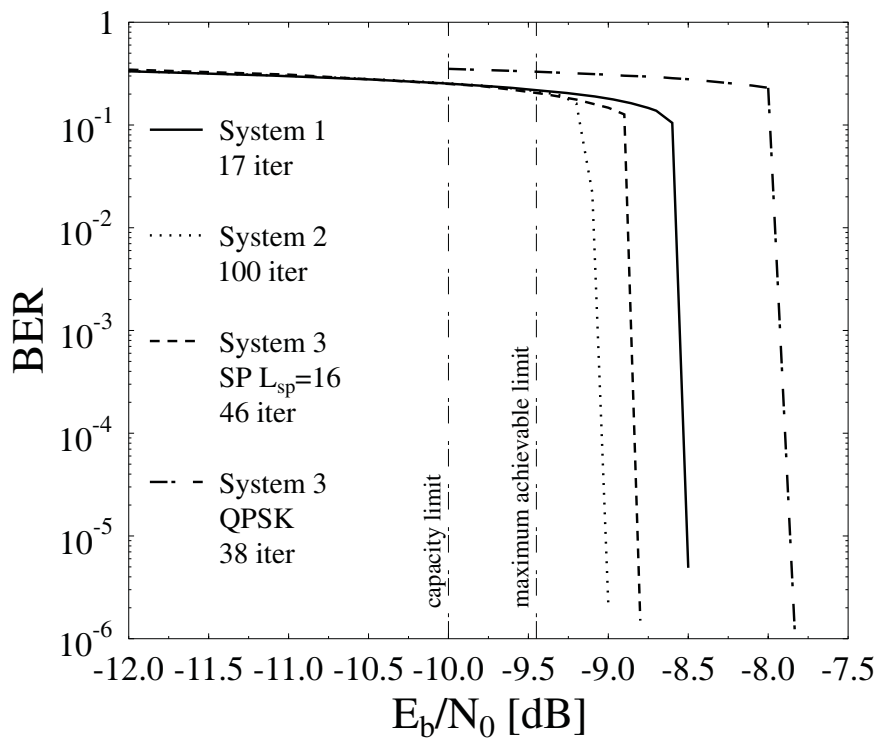

Fig. 5. Performance comparison of the three proposed LSSTC-SP aided systems employing two-stage iteration between an outer code and a URC decoders, as well as three-stage iterative system between an outer RSC, intermediate URC decoders and an SP demapper.

the system derived from the EXIT curves. The discrepancy between the two bandwidth efficiency curves shown in Figure 4 that are calculated using Equation (5) and Equation (6) is due to the fact that Equation (5) was computed for the case where perfect interference cancellation is considered at the receiver. Therefore, Equation (5) constitutes an upper bound on the system's bandwidth efficiency, while Equation (6) constitutes a tighter bound on the maximum achievable bandwidth efficiency of the system considered in this paper.

\section{RESULTS AND Discussion}

In this section, we consider a LSSTC-SP system associated with $L_{s p}=16$ in conjunction with $\left(N_{t}, N_{r}\right)=(4,4)$ antennas. The overall bandwidth efficiency of the system is 2 bits $/ \mathrm{sec} / \mathrm{Hz}$, i.e. the DCMC capacity limit is about $E_{b} / N_{0}=$ $-10 \mathrm{~dB}$, while the maximum achievable rate limit obtained using EXIT charts is at $E_{b} / N_{0}=-9.4 \mathrm{~dB}$ according to Figure 4. Additionally, the presented results correspond to the case, where perfect channel knowledge is considered at the receiver

Figure 5 compares the maximum achievable performance of the three proposed iterative detection aided LSSTC-SP schemes, while employing an interleaver depth of $D=$ 180, 000 bits. Observe that the performance of System 1 and System 3 matches the EXIT chart predictions of Figures 2 and 3 after $I=17$ and $I=46$ iterations, respectively. Hence, System 1 and System 3 perform within $0.9 \mathrm{~dB}$ and $0.6 \mathrm{~dB}$, respectively, from the maximum achievable rate limit. However, System 2 does not closely match the EXIT chart based convergence prediction at $E_{b} / N_{0}=-9.2 \mathrm{~dB}$ even after $I=100$ iterations. This is due to the fact that at $E_{b} / N_{0}=-9.2 \mathrm{~dB}$, the EXIT-tunnel of Figure 2 is narrow and thus requires a significantly higher number of iterations than $I=100$. Thus, after employing $I=100$ decoding iterations System 2 performs within $0.4 \mathrm{~dB}$ from the maximum achievable 


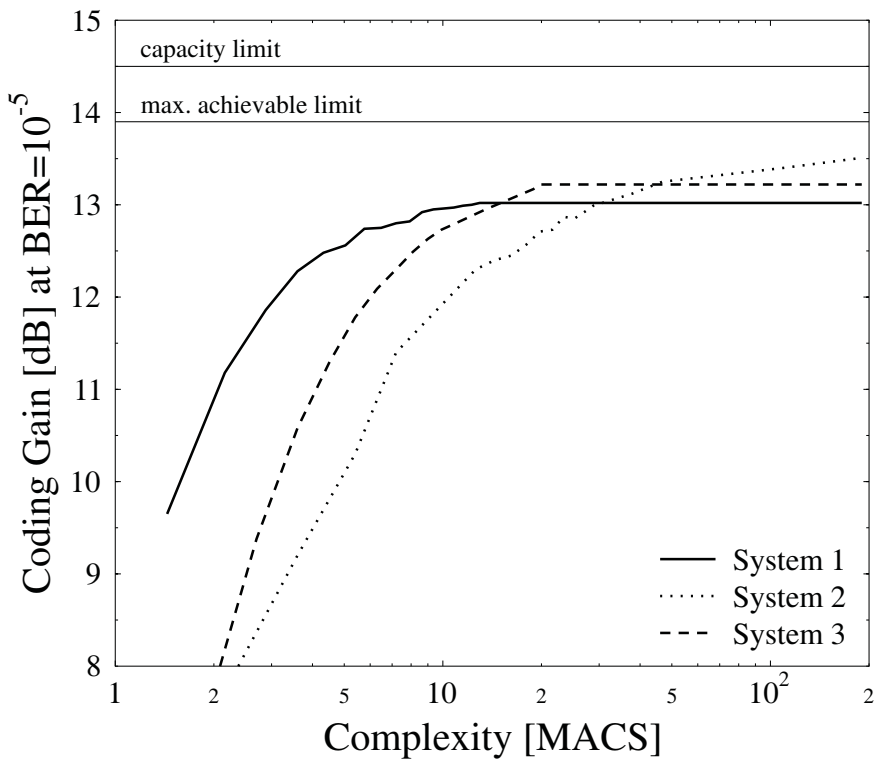

Fig. 6. Comparison of the coding gain at a BER of $10^{-5}$ versus the complexity in MACS of the three proposed LSSTC-SP aided systems

rate limit. Additionally, System 3 employing SP outperforms its counterpart dispensing with SP by $1 \mathrm{~dB}$, as shown in Figure 5.

Finally, Figure 6 shows the coding gain achieved at a BER of $10^{-5}$ for each system versus the detection complexity expressed in terms of Million Add-Compare-Select (MACS) operations. Figure 6 demonstrates that System 1 has the lowest complexity at a distance of $0.9 \mathrm{~dB}$ from the maximum achievable rate limit, where the performance of System 1 approaches an infinitesimally low BER. System 3 is capable of performing equally well in BER terms, while operating $0.3 \mathrm{~dB}$ closer to the maximum achievable rate limit than System 1. However, this is achieved at the cost of almost doubling the complexity. When employing as many as 100 decoding iterations, System 2 becomes capable of performing within $0.4 \mathrm{~dB}$ from the maximum achievable rate limit, although this is achieved at the cost of a complexity that is 20 times that required for operating within $0.9 \mathrm{~dB}$ from the maximum achievable rate limit using System 1.

\section{CONCLUSION}

In this paper, we proposed a novel multi-functional MIMO scheme that combines the benefits of STC, V-BLAST as well as beamforming. The system is also combined with multidimensional SP modulation, facilitating the joint design of the AA's space-time signals. The paper also quantifies the capacity of the LSSTC-SP scheme and proposes a novel procedure for computing an upper limit on the achievable bandwidth efficiency of the system, which is based on EXIT charts. Furthermore, we propose three near-capacity turbo-detected LSSTC-SP schemes, where iterative detection is carried out between the outer code's decoder, the intermediate code's decoder and the LSSTC-SP demapper. Explicitly, the system is capable of operating within $0.9 \mathrm{~dB}, 0.6 \mathrm{~dB}$ and $0.4 \mathrm{~dB}$ from the maximum achievable rate limit. However, to operate within
$0.6 \mathrm{~dB}$ from the maximum achievable rate limit, the system imposes twice the complexity compared to a system operating within $0.9 \mathrm{~dB}$ from the same limit. On the other hand, to operate as close as $0.4 \mathrm{~dB}$ from the maximum achievable rate limit, the system imposes a 20 times higher complexity compared to the one operating within $0.9 \mathrm{~dB}$ from this limit.

\section{REFERENCES}

[1] G. Foschini and M. Gans, "On limits of wireless communications in a fading environment when using multiple antennas," Kluwer Academic Publishers, Wireless Personal Communications, pp. 311-335, 1998.

[2] L. Hanzo, O. Alamri, M. El-Hajjar, N. Wu, Near-Capacity MultiFunctional MIMO Systems: Sphere-Packing, Iteratice Detection and Cooperation. IEEE Press - John Wiley, 2009

[3] L. Hanzo, J. Akhtman, M. Jiang, MIMO-OFDM Turbo-Transceivers for LTE, WIFI and WIMAX. IEEE Press - John Wiley, to appear

[4] P. Wolniansky, G. Foschini, G. Golden, and R. Valenzuela, "V-BLAST: an architecture for realizing very high data rates over the rich-scattering wireless channel," in Proc. International Symposium on Signals, Systems, and Electronics, Pisa, pp. 295-300, Sept. 1998.

[5] V. Tarokh, H. Jafarkhani, and A. Calderbank, "Space-time block codes from orthogonal designs," IEEE Trans. Inform. Theory, vol. 45, no. 5, pp. 1456-1467, 1999.

[6] V. Tarokh, A. Naguib, N. Seshadri, and A. Calderbank, "Combined array processing and space-time coding," IEEE Trans. Inform. Theory, vol. 45, no. 4, pp. 1121-1128, 1999.

[7] M. Tao and R. Cheng, "Generalized layered space-time codes for high data rate wireless communications," IEEE Trans. Wireless Commun., vol. 3, no. 4, pp. 1067-1075, 2004.

[8] L. Hanzo, J. Blogh, and S. Ni, 3G Systems and HSDPA-Style FDD Versus TDD Networking: Smart Antennas and Adaptive Modulation, 2nd ed. John Wiley \& Sons - IEEE Press, Feb. 2008.

[9] G. Jongren, M. Skoglund, and B. Ottersten, "Combining beamforming and orthogonal space-time block coding," IEEE Trans. Inform. Theory, vol. 48, pp. 611-627, Mar. 2002.

[10] W. Su, Z. Safar, and K. Liu, "Space-time signal design for timecorrelated Rayleigh fading channels," in Proc. IEEE International Conference on Communications, vol. 5, pp. 3175-3179, 2003.

[11] O. Alamri, B. Yeap, and L. Hanzo, "A turbo detection and spherepacking-modulation-aided space-time coding scheme," IEEE Trans. Veh. Technol., vol. 56, pp. 575-582, Mar. 2007.

[12] S. Benedetto, D. Divsalar, G. Montorsi, and F. Pollara, "Serial concatenation of interleaved codes: performance analysis, design, and iterative decoding," IEEE Trans. Inform. Theory, vol. 44, pp. 909-926, May 1998.

[13] D. Divsalar, S. Dolinar, and F. Pollara, "Serial concatenated trellis coded modulation with rate-1 inner code," in Proc. IEEE Global Telecommunications Conference (GLOBECOM), vol. 2, (San Francisco, CA), pp. 777-782, 2000.

[14] S. ten Brink, "Designing iterative decoding schemes with the extrinsic information transfer chart," AEÜ International J. Electron. and Commun., vol. 54, pp. 389-398, Nov. 2000.

[15] M. Tüchler, "Convergence prediction for iterative decoding of threefold concatenated systems," in Proc. IEEE Global Telecommunications Conference (GLOBECOM ), vol. 2, pp. 1358-1362, Nov 2002.

[16] J. H. Conway and N. J. Sloane, Sphere Packings, Lattices and Groups. Springer-Verlag, 1999.

[17] R. Gallager, Information Theory and Reliable Communication. New York: Wiley, 1968.

[18] S. X. Ng and L. Hanzo, "On the MIMO channel capacity of multidimensional signal sets," IEEE Trans. Veh. Technol., vol. 55, no. 2, pp. 528-536, 2006.

[19] M. Tüchler, "Design of serially concatenated systems depending on the block length," IEEE Trans. Commun., vol. 52, no. 2, pp. 209-218, 2004.

[20] O. Alamri, J. Wang, S. X. Ng, L. L. Yang, and L. Hanzo, "Near-capacity transceiver design using EXIT-curve fitting: three-stage turbo detection of irregular convolutional coded joint sphere-packing modulation and space-time coding," in Proc. IEEE International Conference on Communications, (Glasgow, Scotland), pp. 4028-4033, June 2007.

[21] M. Tüchler and J. Hagenauer, "EXIT charts of irregular codes," in Proc. Conference on Information Science and Systems, Princeton, NJ, pp. 748753, Mar. 2002. 